\title{
Reproducible gene targeting in recalcitrant Escherichia coli isolates
}

Veerle Derous $^{1 *}$, Francine Deboeck ${ }^{1}$, Jean-Pierre Hernalsteens ${ }^{1}$ and Henri De Greve $e^{2,3}$

\begin{abstract}
Background: A number of allele replacement methods can be used to mutate bacterial genes. For instance, the Red recombinase system of phage Lambda has been used very efficiently to inactivate chromosomal genes in $E$. coli K-12, through recombination between regions of homology. However, this method does not work reproducibly in some clinical $E$. coli isolates.
\end{abstract}

Findings: The procedure was modified by using longer homologous regions ( 85 bp and 500-600 bp), to inactivate genes in the uropathogenic E. coli strain UTI89. An IrhA regulator mutant, and deletions of the lac operon as well as the complete type 1 fimbrial gene cluster, were obtained reproducibly. The modified method is also functional in other recalcitrant $E$. coli, like the avian pathogenic E. coli strain APEC1. The IrhA regulator and lac operon deletion mutants of APEC1 were successfully constructed in the same way as the UTI89 mutants. In other avian pathogenic E. coli strains (APEC3E, APEC11A and APEC16A) it was very difficult or impossible to construct these mutants, with the original Red recombinase-based method, with a Red recombinase-based method using longer (85 bp) homologous regions or with our modified protocol, using 500 - 600 bp homologous regions.

Conclusions: The method using 500-600 bp homologous regions can be used reliably in some clinical isolates, to delete single genes or entire operons by homologous recombination. However, it does not invariably show a greater efficiency in obtaining mutants, when compared to the original Red-mediated gene targeting method or to the gene targeting method with 85 bp homologous regions. Therefore the length of the homology regions is not the only limiting factor for the construction of mutants in these recalcitrant strains.

\section{Background}

The entire genomic sequences of the uropathogenic Escherichia coli strains CFT073, UTI89 and 536 were recently determined [1-3]. At present, functions should be assigned to the relevant open reading frames (ORFs), e.g. by characterizing the appropriate mutant strains.

Precise gene inactivation is an important tool in bacterial genetics. A number of allele replacement methods were developed to mutate bacterial genes. An elegant method, based on homologous recombination, mediated by the Red system of phage Lambda, is used routinely for the construction of deletion mutants in E. coli K-12 [4]. Pathogenic E. coli isolates are often more challenging to manipulate genetically than this laboratory strain. Here we report on the application and validation

\footnotetext{
* Correspondence: Veerle.Derous@vub.ac.be

${ }^{1}$ Viral Genetics Laboratory, Faculty of Science and Bio-engineering Sciences,

Vrije Universiteit Brussel, Pleinlaan 2, B-1050 Brussel, Belgium

Full list of author information is available at the end of the article
}

of a modified Red-mediated gene targeting method, in which 500-600 bp long homology regions were used to delete reproducibly specific genes in E. coli UTI89 (see Figure 1). This modified method allows the directed deletion of any non-essential region - one gene or an entire operon - in the chromosome, by substitution of an antibiotic resistance marker, using Red-mediated homologous recombination. Subsequently, these mutants can easily be converted into non-polar deletions. The described modified method is not only successful in $E$. coli UTI89, but is also effective in other clinical E. coli isolates, like avian pathogenic E. coli (APEC) strains. Its efficiency was compared with the original Red-mediated gene targeting procedure [4] and with another modified Red-mediated gene targeting procedure, in which longer ( $85 \mathrm{bp}$ ) homology regions were used.

\section{Biomed Central}

C 2011 Derous et al; licensee BioMed Central Ltd. This is an open access article distributed under the terms of the Creative Commons Attribution License (http://creativecommons.org/licenses/by/2.0), which permits unrestricted use, distribution, and reproduction in any medium, provided the original work is properly cited. 


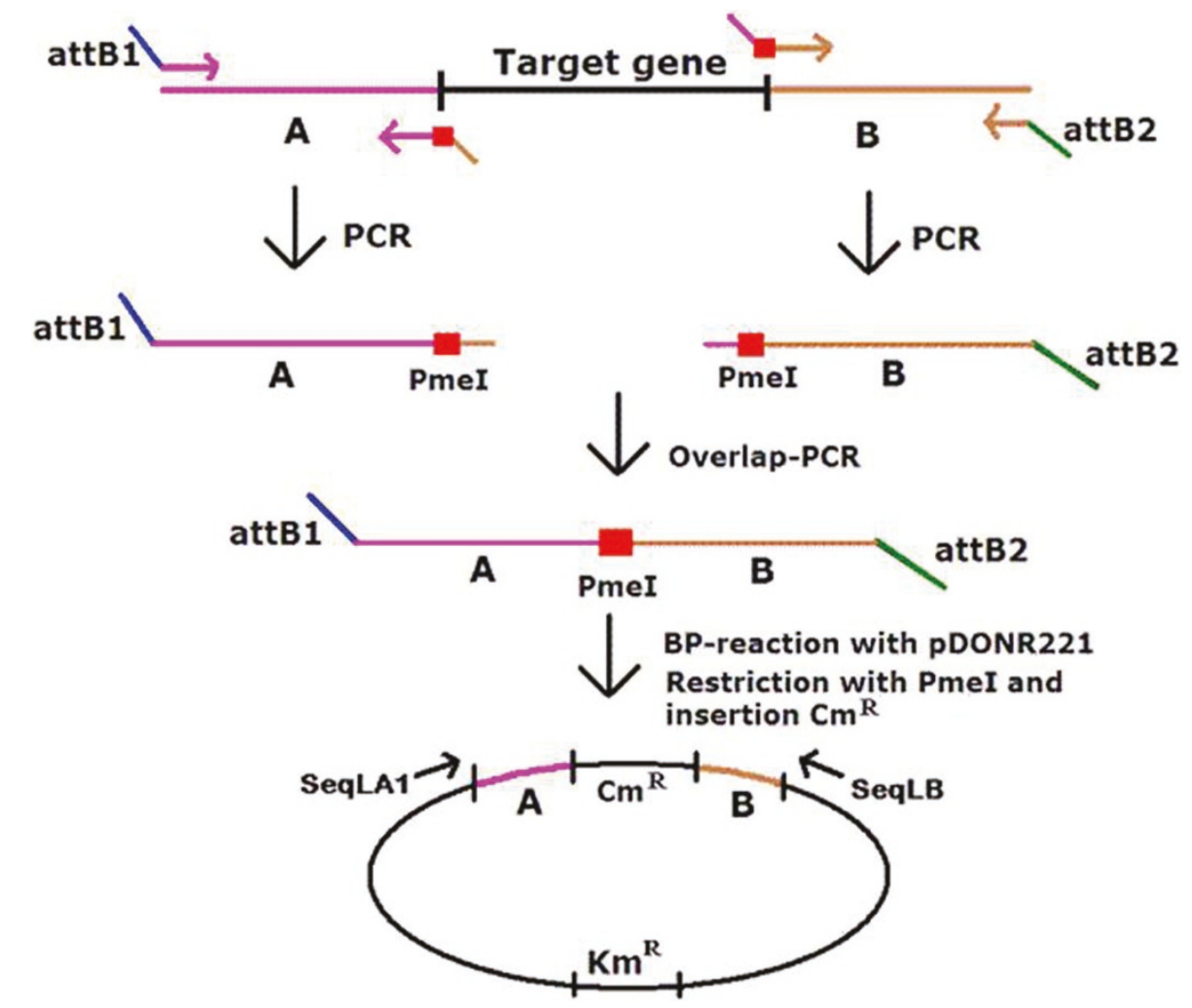

Figure 1 Schematic representation of the construction of specific deletion mutants in E. coli UTI89. In the first step, homology regions A and B, flanking the deletion, were amplified by PCR. Primers were designed in such a way that fragments were generated with an attB-site on one side and with a restriction enzyme site and an overlap region of 25-30 bp on the other side. Overlap-PCR was subsequently carried out with the attB-containing primers, to join regions A and B. The resulting PCR fragment was used in a BP-reaction with the pDONR221 plasmid. This gave rise to a plasmid containing the overlap-PCR fragment. After Pmel restriction of the plasmid, the chloramphenicol resistance marker cat was inserted. A final PCR-fragment was obtained using primers SeqLA1 and SeqLB, which are located on pDONR221. This fragment was electroporated in arabinose-induced E. coli strains harbouring the helper plasmid pKD46 expressing the Red recombinase.

\section{Methods}

Bacterial strains, plasmids and media

The E. coli strain UTI89 (serotype O18:K1:H7) [2] [GenBank:CP000243] is a human cystitis isolate. The E. coli strain APEC1 (serogroup O45) [5] was isolated from the peritoneum of a broiler breeder chicken. The E. coli strain APEC3E (serogroup O78) was isolated from the caecum of a layer chicken and the strains APEC11A and APEC16A (both serogroup O78) were obtained from the heart of layer chickens [6].

The plasmids pKD3 [GenBank: AY048742] and pKD46 [GenBank: AY048746] were described by Datsenko and Wanner [4]. Plasmid pDONR221 was purchased from Invitrogen. The plasmid pEHEC356 [7] was used as a control in the transformation experiments.

Bacteria were grown routinely in LB broth and on LB agar plates [8]. The medium was supplemented with chloramphenicol $(25 \mu \mathrm{g} / \mathrm{ml})$, carbenicilline $(100 \mu \mathrm{g} / \mathrm{ml})$ or kanamycin $(25 \mu \mathrm{g} / \mathrm{ml})$ if needed.

For electroporation, salt-optimized carbon broth (SOC) was used [9].
Phosphate Buffered Saline (PBS) was as described [10].

Modified Red-mediated gene targeting method, using 500-600 bp homology regions (see Figure 1) Amplification and purification of DNA-fragments

Polymerase chain reactions (PCR) were carried out in an Applied Biosystems 2720 Thermal Cycler using Ex Takara DNA Polymerase. Annealing and elongation temperatures were $55^{\circ} \mathrm{C}$ and $68^{\circ} \mathrm{C}$ respectively. Elongation time was 1 minute per $1000 \mathrm{bp}$. The primers used in the PCR reactions are listed in Table 1. PCR-fragments were purified using the Qiaquick PCR Purification Kit (Qiagen). PCR products and purified PCR fragments were analyzed by electrophoresis on $1.0 \%$ agarose gels.

\section{Construction of intermediate gene replacement vectors}

First, two 500-600 bp homology regions ('A' and 'B'), surrounding the targeted sequence, were amplified from total genomic DNA of E. coli UTI89. Primers (see Table 1) are designed in such a way that the PCR-fragments A and B have an overlap of approximately 25-30 bp. After 


\section{Table 1 List of primers}

\begin{tabular}{|c|c|}
\hline Primer & Sequence $\left(5^{\prime}-3^{\prime}\right)$ \\
\hline SeqLA1 & CTCTCGCGTTAACGCTAGCATGGAT \\
\hline SeqLB & GTAACATCAGAGATTTTGAGACAC \\
\hline P1 & GTGTAGGCTGGAGCTGCTTC \\
\hline $\mathrm{P} 2$ & CATATGAATATCCTCCTTAG \\
\hline AttB1LrhA & GGGGACAAGTTTGTACAAAAAAGCAGGCTTACGGCAGATGGACGCCACATCGATT \\
\hline$\underline{\text { LrhAPmel-1 }}$ & AGTATGAGCCGCCAGTAAGTGATAATATATGGTTTAAACTCGACGGACGATAGATAATT \\
\hline LrhAPmel-2 & ATTGTCTCAGGAATTATCTATCGTCCGTCGAGTTTAAACCATATATTATCACTTACTGGCGGCTCA \\
\hline AttB2LrhA & GGGGACCACTITGTACAAGAAAGCTGGGTCTGCTCTTGATGCCGCCTCACCATT \\
\hline LrhA5 & CAGTGATGAGCGATGACTTCAGTG \\
\hline LrhA6 & TAGAAGATTACTTTGCCTAACATA \\
\hline AttB1Lac & GGGGACAAGTTTGTACAAAAAAGCAGGCTTACTACGGCAATGCACTCCTATAA \\
\hline LacPmel-1 & TAACAATTTCACAGGATACAGCTATGGTTTAAACATAAGCAAAATTGCCTGATGCGCTCCGCTT \\
\hline LacPmel-2 & AAGCGGAGCGCATCAGGCAATTTTGCTTATGTTTAAACCATAGCTGTATCCTGTGTGAAATTGTTA \\
\hline AttB2Lac & GGGGACCACTTTGTACAAGAAAGCTGGGTATAAATATCTC ACACGCAATCAAATTCA \\
\hline LacZYA-1 & TTGTTGGGGCGATTCCGCATTTTGAATTTA \\
\hline LacZYA-2 & GTCAGTGGGCTGATCATTAACTAT \\
\hline AttB1Type1 & GGGGACAAGTTTGTACAAAAAAGCAGGCTAT CTCCAGGAAATACACAGTCTGAAA \\
\hline Type1Pmel-1 & TACCTGCATTAGCAATGCCCTGTGA TTTCTGTTTAAACCATCGTTTCCCTTATAATTACAGACGCGCACTA \\
\hline Type1Pmel-2 & TAGTG CGCGTCTGTAATTATAAGGGAAAACGATGGTTTAAACAGAAATCACAGGGCATTGCTAATGCAGGTA \\
\hline AttB2Type1 & GGGGACCACTTTGTACAAGAAAGCTGGGTGTACCAGCG CCAGGTCTGTTCCATGATTT \\
\hline Type1-1 & GAAATATGTTTCCTGGTTITTGGCTTGTAA \\
\hline Type1-2 & ACGACAGACCACACCAGGCCTGCGTCTT \\
\hline Type1DW1 & TATTGCTAACCCAGCACAGCTAGTGCGCGTCTGTAATTATAAGGGAAAACG TTGAGCGATTGTGTAGGCTGGAGCTGCTTC \\
\hline Type1DW2 & GTTIAAGCTTCAGGTAATATTGCGTACCTGCATTAGCAATGCCCTGTGATTTCT TTAGCCATGGTCCATATGAATATCCTCCTTAG \\
\hline LrhADW1 & GTGTGCACAGCATTAACCAGCTCAGTATGAGCCGCCAGTAAGTGATAATA TGTGTAGGCTGGAGCTGCTTC \\
\hline LrhADW2 & CAGCGGCTCGTTITITACACTATTGTCTCAGGAATTATCTATCGTCCGTC CATATGAATATCCTCCTTAG \\
\hline LacZDW1 & GTATGTTGTGTGAAATTGTGAGCGAATAACAATTTCACACAGGATACAGCT TTGAGCGATTGTGTAGGCTGGAGCTGCTTC \\
\hline LacZDW2 & TGAAATTGTAGGCCTGATAAGCGGAGCGCATCAGGCAATTTTGCTTATTTA TTAGCCATGGTCCATATGAATATCCTCCTTAG \\
\hline Type1DWL1 & $\begin{array}{l}\text { CGTAAGCTGACGAATCAGCAGGAATAATCGCTAGGGACCTAAGAATTAGCATGATAATAGCCACTAAGAAATTACTGCGC } \\
\text { TCCATGTGTAGGCTGGAGCTGCTTC }\end{array}$ \\
\hline Type1DWL2 & $\begin{array}{l}\text { TTATCTGGCCTACAAAGGGCTAACGTGCAGGTTITTAGCTTCAGGTAATATTGCGTACCAGCATTAGCAATGTCCTGTGATTTCT } \\
\text { CATATGAATATCCTCCTTAG }\end{array}$ \\
\hline LrhADWL1 & $\begin{array}{l}\text { GGGAGGCACATTACAAATGGAATTGCTTGTTTGTGTGTGCACAGCATTAACCAGCTCAGTATGACCCGCCAGTAAGTGATAATATGT } \\
\text { GTAGGCTGGAGCTGCTTC }\end{array}$ \\
\hline LrhADWL2 & $\begin{array}{l}\text { CTAAAAAAAAGCCGCTGGGGTTTAAAACACCCCCAGCGGCTCGTTTTTTACACTATTGTCTCAGGAATTATCTATCGTCCGTCGAC } \\
\text { CATATGAATATCCTCCTTAG }\end{array}$ \\
\hline LacZDWL1 & $\begin{array}{l}\text { TTAGGCACCCCAGTCTITAC ACTCTATGTG TCCGGCTCGT ATGTTGTGTG AAATTGTGAGCGAATAACAATTTCACACAG GATA } \\
\text { CAGCTTTGAGCGATT GTGTAGGCTG GAGCTGCTTC }\end{array}$ \\
\hline LacZDWL2 & $\begin{array}{l}\text { GCGGCGTGAACACCTTATCC GGCCTACGTA GATCTCTGAA ATTGTAGGCC TGATAAGCGG AGCGCATCAGGCAATTITGC } \\
\text { TTATTIATTAGCCATGGTCCATA TGAATATCCT CCTIAG }\end{array}$ \\
\hline
\end{tabular}

The AttB1 and AttB2 sites are underlined. P1 and P2 sites are italicized.

purification of the PCR-fragments A and B, they were joined by overlap-PCR, which was carried out with the two 'outside' primers containing the attB-sites of the Gateway ${ }^{\circledR}$ cloning system. The Gateway ${ }^{\circledR}$ Technology (Invitrogen) is a universal cloning method providing a rapid and efficient way to move DNA sequences into multiple vector systems. The technology is based on the site-specific recombination system of bacteriophage lambda. This recombination occurs between site-specific attachment (att) sites and is catalyzed by a mixture of enzymes. The components of the lambda recombination system were modified to improve the specificity and efficiency of the system.

The resulting PCR-fragment comprises homology regions $\mathrm{A}$ and $\mathrm{B}$, flanked by $a t t B$-sites, and has a unique 
PmeI restriction enzyme site situated between the two homology regions. The attB sites are used for a site-specific BP-recombination reaction with the attP sites of the pDONR221 vector, using the Gateway ${ }^{\mathbb{R}}$ technology (Invitrogen). After transformation to $\mathrm{CaCl}_{2}$-competent E. coli DH5 $\alpha$ cells [11], clones were selected on LB medium containing kanamycin. Kanamycin-resistant clones were screened by PCR using primers SeqLA1 and SeqLB, which are flanking the insert on pDONR221.

Plasmid DNA was prepared from sequenced clones, using the GeneElute ${ }^{\mathrm{TM}}$ Plasmid Miniprep Kit (Sigma) and cut with restriction enzyme PmeI. Subsequently, the cat gene of plasmid pKD3, flanked by FRT sites, was amplified with primers P1 and P2 [4] and made blunt using T4 DNA polymerase. Ligation of the $1034 \mathrm{bp}$ cat fragment into the PmeI-digested plasmid DNA was performed with the Rapid DNA Ligation Kit (Roche). After transformation into E. coli $\mathrm{DH} 5 \alpha$ competent cells, transformants were selected on chloramphenicol-containing LB medium.

\section{Gene replacement using PCR fragments}

PCR-fragments carrying both homology regions A and $\mathrm{B}$, flanking the P1-FRT-cat-FRT-P2 sequence, were amplified with primers SeqLA1 and SeqLB, using the pDONR221 clones as template. After purification, about $1 \mu \mathrm{g}$ of this DNA was introduced by electroporation (Bio-Rad Gene Pulser apparatus, settings: $25 \mu \mathrm{F}$ and 2.5 $\mathrm{kV}$; Pulse controller set to $200 \Omega$ ) into arabinoseinduced $E$. coli harbouring the Red recombinase system of Lambda, encoded by the helper plasmid pKD46 [4]. After electroporation, cells were immediately resuspended in SOC medium and incubated at $37^{\circ} \mathrm{C}$ for at least one hour. After incubation, aliquots were spread on LB agar plates with chloramphenicol and incubated at $37^{\circ} \mathrm{C}$. Chloramphenicol resistant colonies, that were kanamycin sensitive, were checked by PCR, using primers located outside the flanking homology regions $\mathrm{A}$ and B (see Table 1, primers pairs LrhA5/LrhA6, LacZYA-1/LacZYA-2 or Type1-1/Type1-2), and primers flanking the cat gene (primers P1 and P2). The transformants, that became simultaneously chloramphenicol and kanamycin resistant, were shown to carry the whole pDONR221-derived template plasmid, present in trace amounts in the PCR fragments used for electroporation, instead of an insertion of the cat gene.

\section{Elimination of the antibiotics resistance marker}

The mutants carry the same P1-FRT-cat-FRT-P2 insert as in the original Red recombinase-based method [4]. Therefore the cat gene can be easily removed by expressing the FLP recombinase. This FLP, originating from yeast, recognizes the FRT-sites and mediates a site-specific recombination, so that the antibiotic resistance gene is eliminated leaving the same P1-FRT-P2 scar that has no polar effect on gene expression [4]. The cat gene was eliminated by use of the temperature sensitive helper plasmid pCP20 [4], encoding the FLP recombinase.

For this aim, the plasmid PCP20 was introduced by electroporation in the strain of which the antibiotic resistance marker had to be eliminated. After expression at $28^{\circ} \mathrm{C}$ for 2 hours, the culture was plated on LB medium with carbenicilline $(100 \mu \mathrm{g} / \mathrm{ml})$ and incubated overnight at $28^{\circ} \mathrm{C}$. After purification of the colonies on the same medium, these were purified a second time on LB medium without antibiotics and incubated at $42^{\circ} \mathrm{C}$. The resulting colonies, growing on LB medium but not on $\mathrm{LB}$ with carbenicilline and on LB medium with chloramphenicol, lost the helper plasmid pCP20 and the cat gene.

\section{Original Red-mediated gene targeting method and modified method, using 85 bp homology regions} PCR-fragments, carrying homology regions flanking the type 1, lrhA or lac genes and the cat gene of plasmid pKD3 were purified and about $1 \mu \mathrm{g}$ of this DNA was introduced by electroporation into arabinose-induced $E$. coli strains, harbouring pKD46. Primer pairs LrhADW1/ 2, LacZDW1/2 and Type1DW1/2 (Table 1) were used to amplify the regions flanking $\operatorname{lrh} A$, lac and type 1 genes respectively, for the original Red-mediated gene targeting method [4]. Primer pairs LrhADWL1/2, LacZDWL1/2 and Type1DWL1/2 (Table 1) were used to amplify the regions flanking $\operatorname{lrh} A$, lac and type 1 genes respectively, for the Red-mediated gene targeting method with 85 bp homology regions.

Electroporation for the side-by-side comparison was carried out into the same batch of arabinose-induced cells. To test the transformation efficiency of the strains, the plasmid pEHEC356 was transformed in the same batch of arabinose-induced $E$. coli harbouring the Red recombinase expressing plasmid pKD46.

\section{Phenotypic assays \\ Motility test of $E$. coli strains}

Motility of the E. coli strains was investigated by transferring mutant and wild type colonies onto the same plate containing LB with $0.3 \%$ Select agar (Sigma Aldrich) with a toothpick. After incubation at $30^{\circ} \mathrm{C}$ for $12-16$ hours, the diameter of the colonies was observed [12].

\section{Agglutination of yeast cells}

Adhesion mediated by type 1 fimbriae was tested by agglutination of commercial baker's yeast cells (Saccharomyces cerevisiae) on glass slides [13]. Bacteria were grown statically in $\mathrm{LB}$ broth at $37^{\circ} \mathrm{C}$ for 72 hours. Aliquots of washed bacterial suspensions were incubated with a $2 \%$ yeast suspension in PBS and put on a glass microscope slide. Agglutination was investigated using a stereomicroscope. As a control, 1\% D-mannose (final concentration) was used to inhibit the agglutination mediated by type 1 fimbriae. 


\section{Lactose fermentation assay}

Lactose fermentation was tested on MacConkey medium supplemented with $1 \%$ lactose. Lac mutants grow as white colonies, while wild type $\mathrm{Lac}^{+}$colonies are red.

\section{Statement of ethical approval}

The present study was approved by the Ethical Committee for Animal Experiments of the Vrije Universiteit Brussel (project number 06-219-3) and performed following all national legislation and institutional policies.

\section{Results and discussion}

\section{Gene replacement in E. coli UTI89}

\section{Deletion of the IrhA regulator gene}

The LysR-type transcriptional regulator LrhA (LysR homologue A) controls genes involved in flagellation, motility and chemotaxis [14]. An $\operatorname{lrh} A$ mutant of $E$. coli $\mathrm{K}-12$ showed an increased number of flagellae compared to the wild type. Motility of uropathogenic E. coli (UPEC) plays an important role in colonization of the urinary tract [15]. UPEC presumably utilize flagellum-mediated motility during urinary tract infections to ascend to the upper urinary tract and the kidneys [16]. The transcriptional regulator LrhA also regulates the expression of type 1 fimbriae: inactivation of $\operatorname{lrh} A$ in $E$. coli $\mathrm{K}-12$ results in an increased expression of type 1 fimbriae and increased biofilm formation [13].

Deletion of a single functional gene in E. coli UTI89, by use of the modified Red-mediated gene targeting procedure, in which 500-600 bp long homology regions were used to delete genes, was tested by construction of an UTI89 $\Delta r h A:: \mathrm{Cm}^{\mathrm{R}}$ mutant. The motility of the UTI89 $\Delta$ lrhA $:: \mathrm{Cm}^{\mathrm{R}}$ mutant, in semi-solid LB medium, was much higher than the motility of wild type UTI89 (see Figure 2). This was expected, because Blumer et al. [13] reported increased motility of an $\operatorname{lrh} A$ mutant of $E$. coli MG1655 and UPEC strain 536, due to the increased number of flagellae per cell.

Type 1 fimbriae of $E$. coli mediate the adherence to mannose-containing receptors. Adherence mediated by fimbriae is important for the virulence of uropathogenic E. coli (UPEC), and the expression of type 1 fimbriae is required for colonization of the urinary tract $[17,18]$. Adhesion, mediated by type 1 fimbriae, was monitored by the agglutination of yeast cells. The agglutination caused by the UTI89 $\Delta$ lrh $A:: \mathrm{Cm}^{\mathrm{R}}$ mutant was significantly stronger, compared to the wild type (data not shown). Addition of mannose abolished agglutination, demonstrating that agglutination is mannose-specific and thus depends on the expression of type 1 fimbriae. The UTI89 $\Delta l r h A:: \mathrm{Cm}^{\mathrm{R}}$ mutant also produced more biofilm than wild type UTI89 (data not shown). These

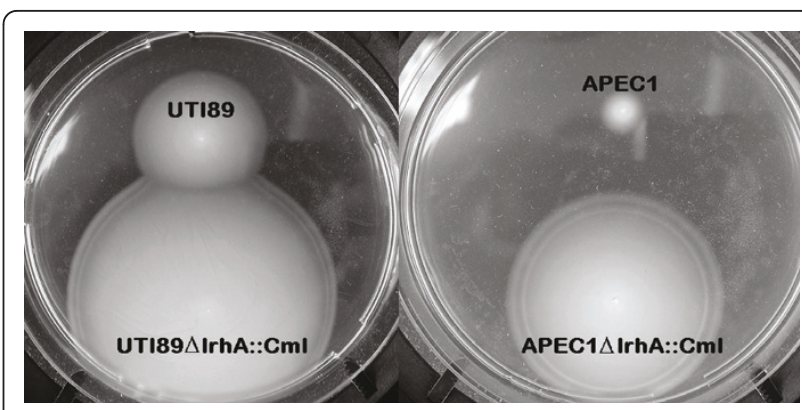

Figure 2 Motility of wild type and IrhA mutant E. coli UTI89 and APEC1. Motility of the E. coli strains was investigated by transferring mutant and wild type colonies onto the same plate containing LB with $0.3 \%$ agar, with a sterile toothpick. After incubation, the diameter of the colonies was compared [12]. The motility of the IrhA mutants is much higher than the motility of the corresponding wild type strains.

results confirm the phenotype of the UTI89 $\Delta / r h A:: \mathrm{Cm}^{\mathrm{R}}$ mutant and show that the described method allows the site-specific deletion of single genes in the E. coli UTI89 genome.

A side-by-side comparison between the original Redmediated gene inactivation method [4], a modified version of the original method with longer (85 bp) homology regions and the described overlap PCR-based method using 500-600 bp homology regions was carried out (See Table 2). UTI89 $\operatorname{lrh} A:: \mathrm{Cm}^{\mathrm{R}}$ mutants were obtained with the three methods.

\section{Deletion of the lac operon}

The lac operon, consisting of three adjacent genes (lacZ, $l a c Y$ and $l a c A$ ) is required for the transport and catabolism of lactose in E. coli [19]. UTI89 $\operatorname{lac} Z Y A:: \mathrm{Cm}^{\mathrm{R}}$ mutants were constructed in the same way as described for the UTI89 $\Delta l r h A:: \mathrm{Cm}^{\mathrm{R}}$ mutant, by use of the modified Red-mediated gene targeting procedure, in which 500-600 bp long homology regions were used. The primer pairs AttB1Lac/LacPmeI-1 and LacPmeI-2/AttB2Lac were used to amplify the homology regions flanking the lac operon. Finally, 18 out of 20 of the chloramphenicol resistant UTI89 tranformants were kanamycin sensitive and produced the expected PCR fragments. All of these UTI89 $\operatorname{lac} Z Y A:: \mathrm{Cm}^{\mathrm{R}}$ grew as white colonies on MacConkey medium supplemented with lactose, while wild type UTI89 produced red colonies. The cat-resistance marker was successfully eliminated by introduction of the pCP20 helper plasmid,

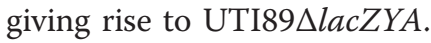

A comparison between the different methods was car-

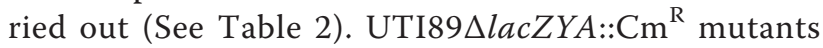
were obtained with the three methods. As was the case for the UTI89 $\Delta l r h A:: \mathrm{Cm}^{\mathrm{R}}$ mutants, the length of the different homology regions used, seemed not to be the determining factor for obtaining mutants. 
Table 2 Side-by-side comparison between the original and modified Red-mediated gene targeting methods

\begin{tabular}{|c|c|c|c|c|c|}
\hline & UT189 & APEC1 & APEC3E & APEC11A & APEC16A \\
\hline \multicolumn{6}{|c|}{ Original Red-mediated gene targeting method } \\
\hline Deletion of type 1 fimbriae cluster & 0 & 0 & 0 & 1 & 0 \\
\hline Deletion of lac operon & 2 & 0 & 0 & 0 & 0 \\
\hline Deletion of IrhA regulator gene & 1 & 0 & 0 & 0 & 0 \\
\hline \multicolumn{6}{|c|}{ Modified Red-mediated gene targeting method using 85 bp long homology regions } \\
\hline Deletion of type 1 fimbriae cluster & 2 & 1 & 0 & 0 & 0 \\
\hline Deletion of lac operon & 1 & 4 & 0 & 0 & 0 \\
\hline Deletion of IrhA regulator gene & 1 & 2 & 2 & 1 & 0 \\
\hline \multicolumn{6}{|c|}{ Modified Red-mediated gene targeting method using $500-600$ bp long homology regions } \\
\hline Deletion of type 1 fimbriae cluster & 2 & 0 & 0 & 0 & 0 \\
\hline Deletion of lac operon & 18 & 2 & 0 & 5 & 0 \\
\hline Deletion of IrhA regulator gene & 1 & 1 & 0 & 0 & 0 \\
\hline
\end{tabular}

The number of chloramphenicol resistant and kanamycin sensitive colonies confirmed by PCR is indicated.

Deletion of the type 1 fimbriae cluster

Adherence of UPEC to the bladder epithelial cells is mediated by type 1 fimbriae and is crucial to prevent washout of the pathogenic bacteria by the flow of urine [17] and for uptake of the bacteria into the bladder epithelial cells. The type 1 fimbriae of UPEC are encoded by the fim gene cluster, consisting of nine genes: fimB, fimE, fim $A$, fimI, fim $C$, fimD, fim, fim $G$ and fim $H$. For the construction of the type 1 fimbrial mutants, primer pairs AttB1Type1/Type1PmeI-1 and Type1PmeI-2/AttB2Type1 were used to amplify the homology regions flanking the type1 gene cluster. Two out of 6 chloramphenicol resistant UTI89 transformants were kanamycin sensitive and were confirmed by PCR. These UTI89 $\triangle$ fimBEAICDFGH:: $\mathrm{Cm}^{\mathrm{R}}$ mutants did not agglutinate yeast cells. The cat-resistance marker was successfully eliminated by introduction of the pCP20 helper plasmid, giving rise to UTI89 $\triangle$ fimBEAICDFGH.

UTI89 $\triangle$ fimBEAICDFGH:: $\mathrm{Cm}^{\mathrm{R}}$ mutants were obtained with both modified versions of the Red-mediated gene targeting method, but no mutant was obtained with the original Red-mediated gene inactivation method (see Table 2).

\section{Gene replacement in avian pathogenic $E$. coli}

We investigated whether the overlap PCR-based method was also functional in the avian pathogenic $E$. coli strain APEC1 (see Table 2). The APEC1 $\Delta l r h A:: \mathrm{Cm}^{\mathrm{R}}$ and APE$\mathrm{C} 1 \triangle$ lacZYA:: $\mathrm{Cm}^{\mathrm{R}}$ mutants were successfully constructed and confirmed by PCR. A motility test with the APEC1 $1 r h A:: \mathrm{Cm}^{\mathrm{R}}$ mutant confirmed that its motility is much higher than the motility of the wild type APEC1 control (see Figure 2). On MacConkey medium supplemented with lactose, APEC1 1 lacZYA:: $\mathrm{Cm}^{\mathrm{R}}$ mutants grew as white colonies, while wild type APEC1 produced red colonies (data not shown). This confirms that the modified Red-mediated gene targeting method, using long (500-600 bp) homology regions can also be applied in this APEC strain. The cat-resistance marker was also successfully eliminated by introduction of the pCP20 helper plasmid, giving rise to APEC1 1 lacZYA.

Mutants were obtained with both modified versions of the Red-mediated gene targeting method, but no mutants were obtained with the original version of the Red-mediated gene inactivation method Although APEC1 showed a 5-times higher number of transformants with the plasmid pEHEC356 than E. coli UTI89, this did not lead to a 5-times larger number of mutants than in E. coli UTI89. Therefore, the efficiency of transformation of these strains is not the determining factor (see Table 2).

We also investigated whether the overlap PCR-based method was functional in the avian pathogenic E. coli strains APEC3E, APEC11A and APEC16A (See Table 2). For APEC3E we only obtained APEC $3 E \Delta l r h A:: \mathrm{Cm}^{\mathrm{R}}$ by use of the Red-mediated procedure with 85 bp homology regions. No other mutants were found in APEC3E by any of the other methods. APEC11A $\Delta \operatorname{lrh} A:: \mathrm{Cm}^{\mathrm{R}}$ mutants were also only obtained with the Red-mediated procedure with $85 \mathrm{bp}$ homology regions, an APEC11A $\Delta$ type $1:: \mathrm{Cm}^{\mathrm{R}}$ mutant was only obtained with the original Red-mediated procedure with 50 bp homology regions and APEC11A $\triangle l a c Z Y A:: \mathrm{Cm}^{\mathrm{R}}$ mutants could only be obtained with the overlap PCR-based procedure with 500-600 bp long homology regions. For APEC16A, it was not possible to construct mutants by any of the three methods used. Nevertheless, transformation of this strain with plasmid DNA was possible at a low frequency (150 colonies were obtained using $1 \mathrm{ng}$ of plasmid pEHEC356 DNA for electroporation). An attempt to inactivate possible restriction-enzyme activity by heating at $50^{\circ} \mathrm{C}$ for $30 \mathrm{~min}$ [20] did not lead to any results in 
the gene targeting and increased the number of pEHEC356 transformants to 200 colonies per ng of plasmid pEHEC356 DNA.

\section{Conclusions}

Methods for disrupting E. coli chromosomal genes, based on an efficient method for E. coli K-12 [4] were tested in clinical UPEC and APEC isolates. Similar Redbased technology has been successfully used for other Gram-negative bacteria, like for example Salmonella [21], Pseudomonas [22], Yersinia [23], Shigella [24] and Vibrio cholerae [25]. Alterations of the gene disruption method are occasionally required $[22,25,26]$. These approaches mostly rely on the expression of a phage recombinase in the bacterial strain. We initially found that deleting specific genes in E. coli UTI89, by the original Red-mediated gene targeting method [4], occurred at a low and variable frequency and was frequently unsuccessful. An alternative strategy for constructing specific mutants, by phage P1-mediated transduction of insertions from E. coli $\mathrm{K}-12$ to $E$. coli UTI89, was also found to be inadequate. The transduction frequency was invariably low and some experiments were unsuccessful. In addition, transduction is impossible when genes are targeted which are not present in the E. coli K-12 genome. We also noticed that a mutant constructed by transduction of an insertion from the Keio collection in E. coli $\mathrm{K}-12$ [12] to UTI89 had a different phenotype than the mutant constructed in UTI89 by the present procedure (data not shown). This is presumably due to the transduction of additional polymorphisms from $E$. coli $\mathrm{K}-12$ to UTI89.

We modified the Red-mediated procedure in such a way that larger regions of homology (500 - $600 \mathrm{bp}$ ) were generated. Our results demonstrate that mutations of specific genes and also deletions of larger chromosomal regions, like for example the lac or the type 1 operon in E. coli UTI89 could be reproducibly obtained. Besides the deletions reported in the present article, mutations in 15 other genes were easily obtained at the first attempt. The method is not only successful in uropathogenic $E$. coli UTI89, but it is also effective in other $E$. coli isolates such as the APEC strain APEC1.

When a final side-by-side comparison was made with the original Red-mediated gene targeting method [4], using 50 and $85 \mathrm{bp}$ long regions of homology, it was found that the length of the homology regions is not the only determining factor for obtaining mutants. In this experiment, the 500-600 bp long homology method was not significantly more efficient in E. coli UTI89 and different APEC strains. This again illustrates our observations on the low reproducibility of Red-mediated gene targeting in clinical isolates. Although the use of 500-600 bp long homology regions increases the reproducibility of the method, other factors, possibly the expression level of the Red proteins at the time of transformation or the efficiency of the electroporation procedure facilitate the targeting in some experiments.

\section{Abbreviations}

Cat: chloramphenicol acetyltransferase; Cm: chloramphenicol; Km: kanamycin.

\section{Acknowledgements}

VD has a Ph.D. grant of the Agency for Innovation by Science and Technology (IWT). The authors are grateful to the Research Council of the Vrije Universiteit Brussel for financial support.

\section{Author details}

${ }^{1}$ Viral Genetics Laboratory, Faculty of Science and Bio-engineering Sciences, Vrije Universiteit Brussel, Pleinlaan 2, B-1050 Brussel, Belgium. ²Structural Biology Brussels, Faculty of Science and Bio-engineering Sciences, Vrije Universiteit Brussel, Pleinlaan 2, B-1050 Brussel, Belgium. ${ }^{3}$ Department of Molecular and Cellular Interactions, VIB, Faculty of Science and Bioengineering Sciences, Vrije Universiteit Brussel, Pleinlaan 2, B-1050 Brussel, Belgium.

\section{Authors' contributions}

VD participated in the design of the study, carried out the DNA manipulation and genetic procedures, wrote the manuscript and drew the figures. FDB performed the experiments on motility, agglutination of yeast cells and lactose fermentation. HDG and J-PH coordinated the study and helped to write the manuscript. All authors read and approved the final manuscript.

Received: 15 February 2011 Accepted: 22 June 2011

Published: 22 June 2011

\section{References}

1. Welch RA, Burland V, Plunkett GD III, Redford P, Roesch P, Rasko DA, Buckles EL, Liou SR, Boutin A, Hackett J, Stroud D, Mayhew GF, Rose DJ, Zhou S, Schwartz DC, Perna NT, Mobley HLT, Donnenberg MS, Blattner FR: Extensive mosaic structure revealed by the complete genome sequence of uropathogenic Escherichia coli. Proc Natl Acad Sci USA 2002, 99:17020-17024.

2. Chen SL, Hung CS, Xu J, Reigstad CS, Magrini V, Sabo A, Blasiar D, Bieri T, Meyer RR, Ozersky P, Armstrong JR, Fulton RS, Latreille JP, Spieth J, Hooton TM, Mardis ER, Hultgren SJ, Gordon JI: Identification of genes subject to positive selection in uropathogenic strains of Escherichia coli: a comparative genomics approach. Proc Natl Acad Sci USA 2006, 103:5977-5982.

3. Hochhut B, Wilde C, Balling G, Middendorf B, Dobrindt U, Brzuszkiewicz E, Gottschalk G, Carniel E, Hacker J: Role of pathogenicity island-associated integrases in the genome plasticity of uropathogenic Escherichia coli strain 536. Mol Microbiol 2006, 61:584-595.

4. Datsenko KA, Wanner BL: One-step inactivation of chromosomal genes in Escherichia coli K-12 using PCR products. Proc Natl Acad Sci USA 2000, 97:6640-6645.

5. Vandemaele F, Bleyen N, Abuaboud O, VanderMeer E, Jacobs A Goddeeris BM: Immunization with the biologically active lectin domain of PapGll induces strong adhesion-inhibiting antibody responses but not protection against avian pathogenic Escherichia coli. Avian Pathol 2006, 35:238-249.

6. Vandekerchove D, Vandemaele F, Adriaensen C, Zaleska M, Hernalsteens JP, De Baets L, Butaye P, Van Immerseel F, Wattiau P, Laevens H, Mast J, Goddeeris B, Pasmans F: Virulence-associated traits in avian Escherichia coli: comparison between isolates from colibacillosis-affected and clinically healthy layer flocks. Vet Microbiol 2005, 15:75-87.

7. Van Gerven N, Derous V, Hernalsteens JP: Expression of in vivo-inducible Salmonella enterica promoters during infection of Caenorhabditis elegans. FEMS Microbiol Lett 2008, 278:236-241.

8. Miller J: A short course in bacterial genetics. A laboratory manual and handbook for E. coli and related bacteria New York: Cold Spring Harbor Laboratory Press; 1992. 
9. Gerhardt P, Muray RGE, Wood WA, Krieg NR: Methods for General and Molecular Bacteriology Washington DC: American Society for Microbiology; 1994.

10. Sambrook J, Russell DW: Molecular Cloning: A Laboratory Manual New York: Cold Spring Harbor Laboratory Press; 2001.

11. Hanahan D, Jessee J, Bloom FR: Plasmid transformation of Escherichia coli and other bacteria. Methods Enzymol 1991, 204:63-113.

12. Niba ETE, Naka Y, Nagase M, Mori H, Kitakawa M: A genome-wide approach to identify the genes involved in biofilm formation in $E$. coli. DNA Research 2007, 14:237-246.

13. Blumer C, Kleefeld A, Lehnen D, Heintz M, Dobrindt U, Nagy G, Michaelis $K$, Emödy L, Polen T, Rachel R, Wendisch VF, Unden G: Regulation of type 1 fimbriae synthesis and biofilm formation by the transcriptional regulator LrhA of Escherichia coli. Microbiol 2005, 151:3287-3298.

14. Lehnen D, Blumer C, Polen T, Wackwitz B, Wendisch VF, Unden G: LrhA as a new transcriptional key regulator of flagella, motility and chemotaxis genes in Escherichia coli. Mol Microbiol 2002, 45:521-532.

15. Wright KJ, Seed PC, Hultgren SJ: Uropathogenic Escherichia coli flagella aid in efficient urinary tract colonization. Infect Immun 2005, 73:7657-7668

16. Lane MC, Alteri CJ, Smith SN, Mobley HLT: Expression of flagella is coincident with uropathogenic Escherichia coli ascension to the upper urinary tract. Proc Natl Acad Sci USA 2007, 104:16669-16674.

17. Martinez JJ, Mulvey MA, Schilling JD, Pinkner JS, Hultgren SJ: Type 1 pilusmediated bacterial invasion of bladder epithelial cells. EMBO J 2000 19:2803-2812.

18. Mulvey MA: Adhesion and entry of uropathogenic Escherichia coli. Cell Microbiol 2002, 4:257-271.

19. Shuman HA, Silhavy TJ: The art and design of genetic screens: Escherichia coli. Nature Reviews Genetics 2003, 4:419-431.

20. Schell J, Glover SW: The effect of heat on host-controlled restriction of phage $\lambda$ in Escherichia coli K(P1). J gen Microbiol 1966, 45:61-72.

21. Husseiny MI, Hensel M: Rapid method for the construction of Salmonella enterica serovar Typhimurium vaccine carrier strains. Infect Immun 2005, 73:1598-1605.

22. Lesic B, Rahme LG: Use of the lambda Red recombinase system to rapidly generate mutants in Pseudomonas aeruginosa. BMC Mol Biol 2008, 9:20.

23. Derbise A, Lesic B, Dacheux D, Ghigo JM, Carniel E: A rapid and simple method for inactivating chromosomal genes in Yersinia. FEMS Immunol Med Microb 2003, 38:113-116.

24. Beloin C, Dorman CJ: An extended role for the nucleoid structuring protein $\mathrm{H}-\mathrm{NS}$ in the virulence gene regulatory cascade of Shigella flexneri. Mol Microbiol 2003, 47:825-838.

25. Yamamoto S, Izumiya H, Morita M, Arakawa E, Watanabe H: Application of lambda Red recombination system to Vibrio cholerae genetics: simple methods for inactivation and modification of chromosomal genes. Gene 2009, 438:57-64.

26. Doublet B, Douard G, Targant H, Meunier D, Madec JY, Cloeckaert A: Antibiotic marker modifications of lambda Red and FLP helper plasmids, pKD46 and pCP20, for inactivation of chromosomal genes using PCR products in multidrug-resistant strains. Journal of Microbiol Meth 2008, 75:359-361.

doi:10.1186/1756-0500-4-213

Cite this article as: Derous et al:: Reproducible gene targeting in recalcitrant Escherichia coli isolates. BMC Research Notes 2011 4:213.

\section{Submit your next manuscript to BioMed Central and take full advantage of:}

- Convenient online submission

- Thorough peer review

- No space constraints or color figure charges

- Immediate publication on acceptance

- Inclusion in PubMed, CAS, Scopus and Google Scholar

- Research which is freely available for redistribution

Submit your manuscript at www.biomedcentral.com/submit 